\title{
Menstrual hygiene practice and its determinants among Adolescent Girls in Sub-Saharan Africa. A systematic review and meta-analysis protocol
}

Etsay Woldu Anbesu ( $\nabla$ etsaywold@gmail.com )

Samara University https://orcid.org/0000-0002-4532-6720

Setognal Birara Aychiluhm

Samara University

\section{Systematic Review}

Keywords: pooled prevalence, determinants, menstrual hygiene practice, adolescent girls, Sub-Saharan Africa

Posted Date: June 25th, 2021

DOI: https://doi.org/10.21203/rs.3.rs-640932/v1

License: (c) (i) This work is licensed under a Creative Commons Attribution 4.0 International License.

Read Full License 


\section{Abstract}

Background: Poor menstrual hygiene practices can lead to gynecological problems, psychosocial stress, and reduced access to school. Menstrual hygiene has not received adequate attention in Sub-Saharan Africa. Moreover, there were fragmented and inconsistent findings. Therefore, this systematic review and meta-analysis aimed to estimate the pooled prevalence of menstrual hygiene practice and identify its associated factors among adolescent girls in Sub-Saharan Africa.

Methods: The protocol for this review had registered at PROSPERO with registration number: CRD42020165628. In this study, the Preferred Reporting Items for Systematic Reviews and MetaAnalyses guideline will be used. Online electronic databases PubMed, Google Scholar, CINAHL and grey literature will be searched to retrieve available studies. Joanna Briggs Institute checklist will be used to assess the quality of the studies. Heterogeneity among studies will be examined using a chi-squared test and I-squared statistic.

To investigate sources of heterogeneity, subgroup analyses and meta-regression will be performed. Sensitivity analysis will be conducted to identify influential studies. Publication bias will be examined by funnel plots and Egger's test. The statistical analysis will be conducted using STATA version-16 software. A random-effect model will be used to estimate the pooled prevalence, and statistical significance will be determined at a $\mathrm{p}$-value of $<0.05$.

Discussion: Poor menstrual hygiene practice affects the health of millions of adolescent girls in developing countries. Currently, there are no synthesis research findings on the overall pooled prevalence of menstrual hygiene practice and its associated factor in Sub-Saharan Africa. Therefore, this systematic review and meta-analysis will be used to inform policy-makers, programmers, planners, clinicians, and researchers to design appropriate strategies.

\section{Introduction}

Menstruation is a physiological process that occurs in most girls during puberty [1]. Good menstrual hygiene management (MHM) practice is described as "women and adolescent girls using a clean menstrual management material to absorb or collect blood that can be modified in privacy as often as necessary for the duration of the menstruation cycle, washing the body as needed with soap and water, and having access to facilities to dispose of used menstrual management materials" [2].

Globally, poor management of menstrual hygiene affects many school girls and women, particularly in developing nations $[3,4]$. In low and middle-income countries, several girls cannot access or afford suitable sanitary materials. Usually, they use low-quality products such as new or old cloth, cotton wool, toilet paper, underwear alone, sponge, or not at all [5-9]. According to a UNICEF report, $10 \%$ of school-age African girls do not attend school during menstruation [10]. Moreover, a study done in five sub-Saharan African countries showed majority of adolescent girls reported a lack of safe, private, and clean toilets with washing facilities at schools [9]. 
Review literature showed that factors associated with poor menstrual hygiene practice were lack of access to clean and effective absorbents, facilities to change, disposal of absorbents, soap, and water in school settings, and lack of privacy $[3,5,11,12]$. Besides, consideration of menstruation as a taboo to discuss with others makes young girls limited knowledge about personal hygiene and develop fear and shame. Even adult women may not be aware of the biological factors of good hygienic practices, difficulties, and preventing them from seeking help [13-15].

Poor menstrual hygiene practices have many consequences. Expos adolescent girls and women to reproductive organ and urogenital infections, psychosocial stress, and reduced opportunities for accessing school and work [12,16-18]. A study done in sub-Saharan African showed that about $49 \%$ of school girls missed four school days monthly due to menstruation [9]. Moreover, studies revealed many school girls suffer concentration, limited participation during class times due to discomfort and dishonor during menstruation [19-21].

In Sub-Saharan Africa countries, menstruation among school-age girls and women is a neglected issue. Adequate attention had not given by the water, sanitation, and hygiene (WASH), education sectors, sexual and reproductive health programs, despite the formal inclusion of menstrual hygiene under reproductive health [22]. Moreover, there were fragmented and inconsistent findings. Thus, this systematic review and meta-analysis aimed to estimate the pooled prevalence of menstrual hygiene practice and identify its associated factors among adolescent girls in Sub-Saharan Africa.

\section{Research Question}

- What is the pooled prevalence of poor menstrual hygiene practice level among adolescent girls in Sub-Saharan Africa?

- What are the determinants of poor menstrual hygiene practice level among adolescent girls in SubSaharan Africa?

\section{Objectives}

- To determine the pooled prevalence of poor menstrual hygiene practice level among adolescent girls in Sub-Saharan Africa.

- To identify determinants of poor menstrual hygiene practice level among adolescent girls in SubSaharan Africa.

\section{Methods}

\section{Study Protocol Registration and Reporting}

The based on the Preferred Reporting Items for Systematic review and Meta-analyses(PRISMA) [23], and Meta-analysis of Observational Studies in Epidemiology (PRISMA-P 2015) checklist [24] (Additional file 1) 
statements to report the findings. The protocol for this review had registered at PROSPERO with registration number: CRD42020165628

\section{PECO search guide}

\section{Population}

Adolescent girl (10-19years) [25]

\section{Exposure}

Predictors or associated factors of poor menstrual hygiene practice. Factors are characteristics or exposures that increase or decrease the likelihood of menstrual hygiene practice among adolescent girls in Sub-Saharan Africa. Those factors include residence, age, maternal educational level, family income, menstrual flow duration, and knowledge on menses.

\section{Comparison}

The reported reference group for each associated factor in each study: adolescent girls residing in urban versus rural, from educated mothers versus no education, with good knowledge on menses versus poor knowledge).

\section{Outcome}

We will include studies that assess the level of poor menstrual hygiene practice and its associated factors among adolescent girls in Sub-Saharan Africa.

\section{Study design and Searching strategy}

This study protocol will use Systematic review and meta-analysis. For the preparation and presentation, preferred Reporting Items for Systematic Reviews and Meta-Analysis will be used online electronic databases PubMed, Google Scholar, CINAHL and grey literature will be used to search articles until April 30,2021 . The searching strategy for the PubMed Online database is added as a supplementary file (Additional file 2). Cross-referencing will also be used to add other studies related to our review. Retrieve studies will be exported to Endnote version 8 reference manager software [26].

Text search terms will be used using Medical Subject Heading (Mesh) keywords searched using PMID. The search strategies will be developed using different Boolean operators. The core search terms and phrases will be (menstruation OR menarche OR menses OR physiology OR psychology OR sexual and reproductive health $\mathrm{OR}$ women health $\mathrm{OR}$ reproductive health $\mathrm{OR}$ hygiene $\mathrm{OR}$ practice $\mathrm{OR}$ hygiene practice OR menstrual hygiene management OR menstrual hygiene OR menstrual health OR Sanitation OR management) AND (menstrual hygiene product OR feminine hygiene product OR sanitary product OR supply \& distribution OR toilet facilities OR standard schools OR school health services OR education OR health education OR risk factors OR prevalence OR quality of life OR adolescent behavior OR standard 
social desirability OR standard OR school absenteeism OR Scholl attendance OR absenteeism) AND (human OR adolescent OR female OR students OR adolescent girls OR young adults OR school girls OR school female) AND (Sub-Saharan Africa OR low income countries OR Developing countries OR poverty)

\section{Study selection and eligibility criteria}

First, duplicate studies will be removed from the Endnote citation manager. Two authors (EW and SB) will independently screen the studies based on inclusion and exclusion criteria. First, relevant articles will be considered based on their title. Secondly, abstracts of the selected titles will be incorporated. Third, the full-text screening will be conducted. In case of articles are not open to access, we will contact the corresponding author. If the authors are not willing to provide full text, the articles will be excluded from the study. Studies that will be approved by both authors in the review processes will be included. During the review of the studies, any disagreement among reviewers will be resolve by discussion and reach a common understanding based on established article selection criteria (Additional file 3). Finally, we will organize a final list of articles for data extraction.

This review will include all observational studies: cross-sectional, analytical cross-sectional, case-control, and cohort studies. Articles published only with the English language, studies done in Sub-Saharan Africa reporting the prevalence of the poor practice of menstrual hygiene and its associated factors among adolescent girls. If a study analyzed knowledge and practice, it will only include if the outcome measures (menstrual hygiene practice) present separately. No restriction will be made to the date of publication. However, citations without abstracts, articles without full text, and data that are difficult to extract will be excluded. Moreover, qualitative studies, adolescent girls whose age group is not well defined, studies published other than the English language, Case reports, conference reports, national survey reports, and expert opinions will be excluded.

\section{Quality assessments}

Articles assessment using their title, abstract, and a full review of the manuscripts will be done before the inclusion of articles in the final meta-analysis. A critical appraisal will be performed by two authors (EW and SB) using the Joanna Briggs Institute Meta-Analysis of Statistics Assessment and Review Instrument (JBI-MASt-ARI) [27]. This instrument includes different questions based on the types of study designs. The tools have Yes and No types of questions and scores will be given ' 1 ' for Yes and ' 0 ' for No responses. Studies $50 \%$ and above of the quality scale will be included and considered for systematic review and meta-analysis (Additional file 4 in $s 1$ files). For any scoring disagreements between the authors, the sources of discrepancy will be investigated through revision. If a difference exists, on the detailed review, the average scores will be calculated. Similarly, each associated factor with the outcome variable will be critically evaluated. A similar cut-off point that we will use for the level of prevalence studies will be used to factors associated with menstrual hygiene practice.

\section{Data extraction and management}


After identifying all eligible articles, two independent reviewers (EW and SB) will extract the relevant data using an organized format on Microsoft Excel Spreadsheet 2016. Pretesting the data extraction form will be done before the beginning of the actual data extraction. If variations of extracted data exist, the phase will be repeated, and then discrepancies between data extractors will be discussed to reach a consensus. For each included article, we will record the author name, year of publication, the study area, study design, study period, sample size, the response rate, the target population, outcome definition, comparison groups, predictors, and overall level of practice. For the level of practice studies, the level of prevalence, the logarithm of the level of prevalence, and standard error (SE) of the logarithm of the level prevalence will be calculated. Similarly, for associated factors, OR, logarithms of OR, and SE of the logarithms of OR will be calculated. For any difficulties that might be encountered during data extraction, communication will be made with the corresponding author.

\section{Outcome measurement}

Good menstrual hygiene management (MHM) practice is described as "women and adolescent girls using a clean menstrual management material to absorb or collect blood that can be modified in privacy as often as necessary for the duration of the menstruation cycle, washing the body as needed using soap and water, and having access to facilities to dispose of used menstrual management materials"[2]. There is a variation in the number of questions to measure menstrual hygiene practice in different studies. Therefore, the menstrual hygienic practices will be evaluated using "Yes" or "No" questions. The response of each item will be scored as " 1 " for correct answers and " 0 " for false. The total sum score of $50 \%$ and above will be considered as having a good menstrual hygiene practice [28, 29].

\section{Data synthesis and analysis}

The extract data will be imported into STATA version 14software. A narrative description of the study population will be done, and tables and figures will be used to summarize the results.

A random-effect model will be used to estimate the overall pooled prevalence of menstrual hygiene practice among adolescent girls in Sub-Saharan Africa [30]. The Freeman Tuckey variant of the arcsine square root transformation of proportions will be used to avoid variance variability when controlling proportions close to one[31, 32]. We will assess heterogeneity by using the chi-squared test on Cochran's $\mathrm{Q}$ statistic with a $5 \%$ level of statistical significance [33] and $\mathrm{I}^{2}$ statistic test [34]. Assuming $\mathrm{I}^{2}$ values of $25 \%, 50 \%$, and $75 \%$ is representative of low, moderate, and high heterogeneity respectively. If the heterogeneity is significant $\left(\mathrm{I}^{2}>75 \%\right)$, and $\mathrm{p}$-value $<0.05$ will be declared as the presence of heterogeneity. Hence, subgroup analyses and meta-regression will be performed to investigate sources of heterogeneity.

Sensitivity analysis will be done to see the effect of a particular study on the overall estimation. Besides, the time-trend analysis will be conducted to check whether variations through time are observed [33]. Publication bias will be examined by the visual inspection of funnel plots [35] and Egger's test [36]. A pvalue $<0.10$ will be considered indicative of statistically significant publication bias. If evidence of publication bias present, the trim-and-fill (Duval and Tweedie's) method will be performed [37]. The 
existence of an association between the factors and menstrual hygiene practice will be estimated based on the effect size. Then, the statistical significance level will be declared at a p-value of less than 0.05 .

\section{Discussion}

This systematic review and meta-analysis protocol aim to synthesize research findings on the overall pooled prevalence of menstrual hygiene practice and its associated factor in Sub-Saharan Africa. Poor menstrual hygiene practice affects the health of millions of adolescent girls, particularly in developing countries. Studies in developing countries have reported that more than $50 \%$ of girls have inadequate menstrual hygiene management (MHM) practices, with higher proportions in rural areas [38, 39]. Millions of adolescent girls suffer from complex problems, though good menstrual hygienic management practices can have many benefits [40].

A review of qualitative and quantitative research findings showed that poor menstrual hygiene practice affects school absenteeism, distraction, and detachment, poor psychosocial consequences, feelings of shame, fear, fear of stigma, and anxiety [41-44]. Moreover, most young age women don't have the comfort to discuss menses, as it is has a social taboo and could not have access to gain adequate information [45]. It is the overlooked opportunity to address the level of menstrual hygiene practice among girls earlier from their adolescent age in most low-income countries [46]. Thus, effective menstrual hygiene management practice is essential for women and girls to participate in society with dignity and comfort [4]. Consequently, it remains important to assess the level of menstrual hygiene practice and its associated factors in Sub-Saharan Africa to inform the development of appropriate programs and policies that will have an impact in reducing poor menstrual hygiene practice in the region.

The finding from this systematic review will help national and non-governmental organizations in the health and education sector of each country of the region to emphasize the main factors that drive poor menstrual hygiene practice. Moreover, the finding will increase accessibility and availability of services to improve the health status of adolescent girls. Therefore, estimating the overall level of pooled prevalence of menstrual hygiene practice and its associated factors in Sub-Saharan African using high-level evidence will be used to inform policy-makers, health programmers, education sectors, clinicians, decision making, and researchers.

This study protocol has the following limitation. Heterogeneity is common as we will consider varieties of study designs and from different geographic areas of Sub-Saharan African countries. Articles published other than the English language will not be considered. However, there might be articles published in other languages. Moreover, this protocol considers only observational studies design and excludes randomized clinical trials and quasi-experimental studies.

\section{Declarations}

\section{Author contribution}


EW and SB conceived and designed the systematic review and meta-analysis. EW drafted the protocol manuscript. EW and SB extensively reviewed and incorporated inputs in the protocol manuscript development. Both authors read and approved the final version of the protocol manuscript.

\section{References}

1. Winkler, I.T. and V. Roaf, Taking the bloody linen out of the closet: menstrual hygiene as a priority for achieving gender equality. Cardozo JL \& Gender, 2014. 21: p. 1.

2. Biran, A., V. Curtis, O.P. Gautam, et al., Background paper on measuring WASH and food hygiene practices: definition of goals to be tackled post 2015 by the joint monitoring programme. London Sch Hyg Trop Med, 2012. 2012: p. 81.

3. Sommer, M., B.A. Caruso, M. Sahin, et al., A time for global action: addressing girls' menstrual hygiene management needs in schools. PLoS medicine, 2016. 13(2): p. e1001962.

4. Sommer, M. and M. Sahin, Overcoming the taboo: advancing the global agenda for menstrual hygiene management for schoolgirls. American journal of public health, 2013. 103(9): p. 1556-1559.

5. Enzler, D.M., Knowledge, attitudes and practices concerning Menstrual Hygiene Management (MHM) of adolescents in rural primary schools in Malawi. 2019.

6. Fisseha, M.A., Y. Kebede, and H.Y. Yeshita, Menstrual hygiene practice and associated factors among secondary school girls in Wegera District, Northwest Ethiopia; a cross-sectional study. Computational Biology and Bioinformatics, 2017. 5(1): p. 6-11.

7. Geertz, A., An opportunity to address menstrual health and gender equity. 2016: FSG.

8. Kuhlmann, A.S., K. Henry, and L.L. Wall, Menstrual hygiene management in resource-poor countries. Obstetrical \& gynecological survey, 2017. 72(6): p. 356.

9. Tamiru, S., K. MAMO, P. ACIDRIA, et al., Towards a sustainable solution for school menstrual hygiene management: cases of Ethiopia, Uganda, South-Sudan, Tanzania, and Zimbabwe. Waterlines, 2015: p. 92-102.

10. Education, P., Menstrual Hygiene Management-UNESCO Digital Library. United Nations Educational, Scientific and Cultural Organization: Paris, France, 2014.

11. Mason, L., E. Nyothach, K. Alexander, et al., 'We keep it secret so no one should know'-A qualitative study to explore young schoolgirls attitudes and experiences with menstruation in rural Western Kenya. PloS one, 2013. 8(11): p. e79132.

12. Phillips-Howard, P.A., B. Caruso, B. Torondel, et al., Menstrual hygiene management among adolescent schoolgirls in low-and middle-income countries: research priorities. Global health action, 2016. 9(1): p. 33032.

13. Chothe, V., J. Khubchandani, D. Seabert, et al., Students' perceptions and doubts about menstruation in developing countries: a case study from India. Health promotion practice, 2014. 15(3): p. 319326. 
14. House, S., T. Mahon, and S. Cavill, Menstrual hygiene matters: a resource for improving menstrual hygiene around the world. Reproductive Health Matters, 2013. 21(41): p. 257-259.

15. Sommer, M., C. Sutherland, and V. Chandra-Mouli, Putting menarche and girls into the global population health agenda. Reproductive health, 2015. 12(1): p. 1-3.

16. Baker, K., A. Dutta, T. Swain, et al., Menstrual Hygiene Practices, WASH Access and the Risk of Urogenital Infection in Women from Odisha, India: Supporting Information. 2015.

17. Hulland, K.R., R.P. Chase, B.A. Caruso, et al., Sanitation, stress, and life stage: a systematic data collection study among women in Odisha, India. PloS one, 2015. 10(11): p. e0141883.

18. Mathiyalagen, P., B. Peramasamy, K. Vasudevan, et al., A descriptive cross-sectional study on menstrual hygiene and perceived reproductive morbidity among adolescent girls in a union territory, India. Journal of family medicine and primary care, 2017. 6(2): p. 360.

19. Boosey, R., G. Prestwich, and T. Deave, Menstrual hygiene management amongst schoolgirls in the Rukungiri district of Uganda and the impact on their education: a cross-sectional study. The Pan African Medical Journal, 2014. 19.

20. Hennegan, J., C. Dolan, M. Wu, et al., Measuring the prevalence and impact of poor menstrual hygiene management: a quantitative survey of schoolgirls in rural Uganda. BMJ open, 2016. 6(12).

21. Tegegne, T.K. and M.M. Sisay, Menstrual hygiene management and school absenteeism among female adolescent students in Northeast Ethiopia. BMC public health, 2014. 14(1): p. 1-14.

22. Ssewanyana, D. and B.K.Y. Bitanihirwe, Menstrual hygiene management among adolescent girls in sub-Saharan Africa. Global health promotion, 2019. 26(1): p. 105-108.

23. Moher, D., A. Liberati, J. Tetzlaff, et al., Preferred reporting items for systematic reviews and metaanalyses: the PRISMA statement. PLoS medicine, 2009. 6(7): p. e1000097.

24. Shamseer, L., D. Moher, M. Clarke, et al., Preferred reporting items for systematic review and metaanalysis protocols (PRISMA-P) 2015: elaboration and explanation. Bmj, 2015. 349.

25. Nation, U., United Nations Department of Economic and Social Affairs (UNDESA) 2007.

26. Bramer, W. and P. Bain, Updating search strategies for systematic reviews using EndNote. Journal of the Medical Library Association: JMLA, 2017. 105(3): p. 285.

27. Munn, Z., C. Tufanaru, and E. Aromataris, JBI's systematic reviews: data extraction and synthesis. AJN The American Journal of Nursing, 2014. 114(7): p. 49-54.

28. Supply, W. and S.C. Council, Celebrating Womanhood: How better menstrual hygiene management is the path to better health, dignity and business. Malaysia: WSSCC, 2013.

29. Upashe, S.P., T. Tekelab, and J. Mekonnen, Assessment of knowledge and practice of menstrual hygiene among high school girls in Western Ethiopia. BMC women's health, 2015. 15(1): p. 1-8.

30. Berkey, C.S., D.C. Hoaglin, F. Mosteller, et al., A random-effects regression model for meta-analysis. Statistics in medicine, 1995. 14(4): p. 395-411.

31. Lin, L. and C. Xu, Arcsine-based transformations for meta-analysis of proportions: Pros, cons, and alternatives. Health Science Reports, 2020. 3(3): p. e178. 
32. Nyaga, V.N., M. Arbyn, and M. Aerts, Metaprop: a Stata command to perform meta-analysis of binomial data. Archives of Public Health, 2014. 72(1): p. 1-10.

33. Cooper, H., L.V. Hedges, and J.C. Valentine, The handbook of research synthesis and meta-analysis. 2019: Russell Sage Foundation.

34. Higgins, J.P. and S.G. Thompson, Quantifying heterogeneity in a meta-analysis. Statistics in medicine, 2002. 21(11): p. 1539-1558.

35. Liu, J.L., The role of the funnel plot in detecting publication and related biases in meta-analysis. Evidence-based dentistry, 2011. 12(4): p. 121-122.

36. Egger, M., G.D. Smith, M. Schneider, et al., Bias in meta-analysis detected by a simple, graphical test. Bmj, 1997. 315(7109): p. 629-634.

37. Duval, S. and R. Tweedie, Trim and fill: a simple funnel-plot-based method of testing and adjusting for publication bias in meta-analysis. Biometrics, 2000. 56(2): p. 455-463.

38. Adinma, E.D. and J. Adinma, Perceptions and practices on menstruation amongst Nigerian secondary school girls. African journal of reproductive health, 2008. 12(1): p. 74-83.

39. Bairwa, M., M. Rajput, and S. Sachdeva, Modified Kuppuswamy's socioeconomic scale: social researcher should include updated income criteria, 2012. Indian journal of community medicine: official publication of Indian Association of Preventive \& Social Medicine, 2013. 38(3): p. 185.

40. Nair, M., D. Chacko, M.R. Darwin, et al., Menstrual disorders and menstrual hygiene practices in higher secondary school girls. The Indian Journal of Pediatrics, 2012. 79(1): p. 74-78.

41. Crichton, J., J. Okal, C.W. Kabiru, et al., Emotional and psychosocial aspects of menstrual poverty in resource-poor settings: a qualitative study of the experiences of adolescent girls in an informal settlement in Nairobi. Health care for women international, 2013. 34(10): p. 891-916.

42. Grant, M., C. Lloyd, and B. Mensch, Menstruation and school absenteeism: evidence from rural Malawi. Comparative education review, 2013. 57(2): p. 260-284.

43. Lloyd, C.B. and J. Young, New lessons: The power of educating adolescent girls-A girls count report on adolescent girls. 2009.

44. Sommer, M., N. Ackatia-Armah, S. Connolly, et al., A comparison of the menstruation and education experiences of girls in Tanzania, Ghana, Cambodia and Ethiopia. Compare: A Journal of Comparative and International Education, 2015. 45(4): p. 589-609.

45. Wall, L.L., S. Belay, A. Bayray, et al., A community-based study of menstrual beliefs in Tigray, Ethiopia. International Journal of Gynecology \& Obstetrics, 2016. 135(3): p. 310-313.

46. Chandra-Mouli, V. and S.V. Patel, Mapping the knowledge and understanding of menarche, menstrual hygiene and menstrual health among adolescent girls in low-and middle-income countries. Reproductive health, 2017. 14(1): p. 1-16.

\section{Declaration}

\section{Competing interests}


The authors declare no competing interests.

\section{Supplementary Files}

This is a list of supplementary files associated with this preprint. Click to download.

- Additionalfile1.doc

- Additionalfile2.docx

- Additionalfile3.docx

- Additionalfile4.docx 